\title{
Parlamentsplenum und Bürgerkonferenz: Was leisten sie auf dem Weg zur politischen Entscheidung?
}

\author{
Claudia Landwehr und Katharina Holzinger
}

Eine zentrale Annahme deliberativer Demokratietheorien lautet, dass politische Präferenzen durch kommunikative Interaktion gebildet und transformiert werden. ${ }^{1}$ Sie verändern sich zum Besseren, so die Annahme, wenn ein Diskurs hinreichend machtfrei und inklusiv ist. Von den Präferenzen, die aus einem Diskurs hervorgehen, wird erwartet, dass sie besser informiert sind und, im Sinne John Harsanyis ${ }^{2}$, „ethisch“ statt „subjektiv“ sind. Darüber hinaus wird unterstellt, dass Präferenzen auf einen Konsens hin konvergieren, dessen Inhalt mit höherer Wahrscheinlichkeit gerecht und rational ist als das Ergebnis einer Präferenzaggregation ohne Deliberation.

Allerdings sind mehrere Elemente dieser theoretischen Grundannahmen unzureichend theoretisiert, und auch an empirischen Belegen mangelt es weitgehend. Eines der Probleme der deliberativen Theorie ist das Fehlen einer Theorie der Präferenztransformation, die erklären könnte, wie und warum genau Kommunikation auf Präferenzen wirkt. ${ }^{3}$ Ein weiteres, in mancherlei Hinsicht hiermit zusammenhängendes Problem ist die Tatsache, dass Deliberation zwar als Modus der Interaktion einvernehmlich gepriesen wird, es aber relativ wenig Konsens darüber gibt, wie sie institutionalisiert werden könnte. ${ }^{4}$ Ein zentrales Problem ihrer empirischen Erforschung liegt im Fehlen einer Übereinkunft darüber, wie die Theorie zu operationalisieren und welche Hypothesen zu bestätigen wären, um Belege für ihre Grundannahmen zu gewinnen. Es erscheint daher notwendig, explizite Hypothesen über die Wirkungen institutioneller Parameter auf Interaktionsmodi sowie über die Wirkungen dieser Interaktionsmodi auf Präferenzbildung und -transformation zu formulieren. Während es

1 Einer der ersten, der den Begriff „deliberative Demokratie“ verwendet hat, war Joseph M. Bessette, Deliberative Democracy: The Majority Principle in Republican Government, in: Robert A. Goldwin / William A. Schambra (Hrsg.), How Democratic is the Constitution?, Washington 1980. Unterschiedliche Modelle deliberativer Demokratie haben etwa Amy Gutmann / Dennis Thompson, Democracy and Disagreement, Cambridge 1996; James Bohman, Public Deliberation. Pluralism, Complexity, and Democracy, Cambridge 1996; John Dryzek, Deliberative Democracy and Beyond, Oxford 2000; Robert E. Goodin, Reflective Democracy, Oxford 2003, vorgeschlagen. Sehr gute Sammelbände liegen von James Bohman / William Rehg (Hrsg.), Deliberative Democracy, Cambridge 1997; Jon Elster (Hrsg.) Deliberative Democracy, Cambridge 1998; Stephen Macedo (Hrsg.), Deliberative Politics. Essays on ,Democracy and Disagreement', Oxford 1999; James S. Fishkin / Peter Laslett (Hrsg.), Debating Deliberative Democracy, Malden 2003 vor.

2 John C. Harsanyi, Cardinal Welfare, Individualistic Ethics, and Interpersonal Comparisons of Utility, in: Journal of Political Economy, 63. Jg. (1955), H. 4, S. $309-321$.

3 Ein Pionier im Bereich der empirischen Forschung über Präferenztransformation war James $S$. Fishkin, The Voice of the People: Public Opinion and Democracy, New Haven 1991. Eine jüngere Arbeit zum Thema stammt von Kasper M. Hansen, Deliberative Democracy and Opinion Formation, Odense 2004.

4 Zur Verwendung partizipativer Verfahren zur Institutionalisierung deliberativer Demokratie gibt es ein Handbuch von John Gastil / Peter Levine (Hrsg.), The Deliberative Democracy Handbook, San Francisco 2005. Zur Verwendung deliberativer Demokratie im Institutionendesign für gespaltene Gesellschaften siehe Ian O'Flynn, Deliberative Democracy in Divided Societies, Edinburgh 2006. 
dem übergreifenden Forschungsprogramm der deliberativen Theorie um das Verhältnis zwischen politischen Institutionen, Kommunikation und Akteursmotivation geht, können hier nur einzelne Aspekte davon herausgegriffen werden. Dabei wird ein "disaggregierender" Ansatz der empirischen Deliberationsforschung im Sinne Dennis Thompsons ${ }^{5}$ verfolgt beziehungsweise ein „middle-range theory building“, wie Diana C. Mutz es fordert. ${ }^{6}$

Im theoretischen Teil werden zwei Bedingungen identifiziert, unter denen kommunikative Interaktion Wirkung auf Akteurspräferenzen haben kann: Diskursivität und Koordinativität. Durch die Anordnung dieser zwei Eigenschaften in einer Matrix werden vier idealtypische Interaktionsmodi gewonnen, für die Hypothesen über die jeweilige Wahrscheinlichkeit von Präferenztransformation formuliert werden. Geht man der Frage nach, ob und wie diese Interaktionsmodi institutionalisiert werden können, so erscheinen zwei institutionelle Kontexte als maximal förderlich, respektive maximal hinderlich für Präferenztransformation: Ersterer findet sich im Modell der Bürger- beziehungsweise Konsensuskonferenz verwirklicht, letzterer in der typischen Plenardebatte in parlamentarischen Regierungssystemen, wo der überwiegende Teil der legislativen Arbeit von Ausschüssen und Fraktionen übernommen wird.

Im empirischen Teil wird eine vergleichende Analyse einer parlamentarischen Debatte und einer Bürgerkonferenz präsentiert, die sich mit demselben Konflikt befasst haben: der Entscheidung über den Import embryonaler Stammzellen nach Deutschland. Dieser spezifische Konflikt ist deshalb interessant, weil die politischen Parteien ebenso wie soziale Gruppen in der Frage tief gespalten waren und die Entscheidung deshalb nicht entlang einer einfachen Links-Rechts-Dimension erfolgte. Entsprechend wurde die Fraktionsdisziplin im Bundestag aufgehoben, was Abgeordneten erlaubte, sich ohne intrafraktionelle Abstimmungsprozesse und -notwendigkeiten eine eigene Meinung zu bilden, und Präferenztransformation zumindest prinzipiell möglich machte. In einem ersten Schritt werden Transkripte aus beiden Foren mit der Methode der Sprechaktanalyse untersucht, im zweiten Schritt Auftreten und Richtung von Präferenzänderungen. Obgleich die Analyse von nur zwei Foren offensichtlich als Hypothesentest unzureichend ist, untermauern die Ergebnisse die theoretischen Überlegungen und verweisen im Sinn einer explorativen Studie auf Ansatzpunkte für die weitere Forschung zum Thema.

\section{Theorie und Hypothesen: eine Typologie von Interaktionsmodi}

Eine zentrale Annahme der Theorie deliberativer Demokratie lautet, dass Präferenzen politischen Diskursen und Entscheidungsverfahren endogen sind, in diesen gebildet und transformiert werden. Der Austausch von Gründen, der im Mittelpunkt der deliberativen Demokratie steht, kann politische Entscheidungen nur dann leiten, wenn, wie Jürgen Habermas feststellt, Gründe auch zugleich Motive sind ${ }^{7}$ beziehungsweise die politischen Präferenzen der Akteure beeinflussen.

5 Dennis Thompson, Deliberative Democratic Theory and Empirical Political Science, in: Annual Review of Political Science, 11. Jg. (2008), S. 497 - 520, S. 509.

6 Diana C. Mutz, Is Deliberative Democracy a Falsifiable Theory?, in: Annual Review of Political Science, 11. Jg. (2008), S. $521-538$, S. 522.

7 Vgl. Jürgen Habermas, Faktizität und Geltung, Frankfurt am Main 1994, S. 188. 
Hier werden politische Präferenzen als vergleichende Bewertung von Handlungsergebnissen verstanden, die eine kognitive und eine volitive Komponente haben. Letztere betrifft die Wünschbarkeit von Weltzuständen, erstere die instrumentelle Eignung von Optionen, diese herbeizuführen. Bei der Analyse politischer Entscheidungsprozesse ist es sinnvoll, Präferenzen als über politische Handlungsoptionen (Policy-Optionen) definiert anzusehen.

Im Hinblick auf die kognitive Komponente politischer Präferenzen ist der Fall für ihre Transformierbarkeit relativ klar: Kognitive Einstellungen (Überzeugungen oder Annahmen) verlieren ihren Zweck, wenn sie für neue Informationen und Argumente nicht offen sind. Ein erheblicher Teil der Konflikte zwischen ethischen Präferenzen hängt in der Tat mit konfligierenden Annahmen über die instrumentelle Qualität von Politikoptionen zur Erreichung geteilter Ziele zusammen. Dies ist der mehr oder weniger unstrittige und vielleicht auch weniger interessante Fall. Weitaus umstrittener und zugleich interessanter ist die Transformation der volitiven Komponente von Präferenzen. Zunächst scheint klar, dass sich Ziele, Werte und Interessen unmöglich ständig verändern können. Wenn ein Ziel nicht über einen gewissen Zeitraum stabil und für die Entscheidungen eines Akteurs effektiv bleibt, besteht wenig Sinn darin, es überhaupt anzunehmen und zu verfolgen. Und wenn Normen und Werte sich beständig änderten, dann könnten sie nicht als allgemeine Prinzipien Handeln anleiten. Wenn Deliberation Präferenzen gemeinwohl-orientierter oder gerechter machen soll, müssen jedoch auch volitive Einstellungen transformierbar sein. Die deliberative Demokratietheorie geht davon aus, dass sie durch Auseinandersetzung mit neuen praktischen Handlungsgründen verändert werden. Wir gehen davon aus, dass ihre Transformation in der Regel auf einer neuen Gewichtung und Aggregation konkurrierender Gründe (Ziele, Normen, Werte) beruht.

Nimmt man den Schritt von der individuellen Entscheidungsfindung zu den Herausforderungen kommunikativer Interaktion und kollektiver Entscheidung, so besteht die Verbindung zwischen den beiden Ebenen in der Forderung nach Rechtfertigung von Präferenzen, also nach Gründen. Individuen beteiligen sich an Diskursen, um diese Gründe zu prüfen und zu verbessern, und Rechtfertigungsdiskurse bilden und verändern Präferenzen. Der Rechtfertigungsdruck, der in kommunikativer Interaktion ausgeübt wird, lässt sich mit einem allgemeinen Prinzip der Kooperation - oder Reziprozität - erklären, das für den Sprachgebrauch konstitutiv ist. ${ }^{8}$ Ein solches hilft, Habermas' Feststellung einzuordnen, dass Verständigung der „inhärente Telos der menschlichen Sprache“ sei.?

Obwohl Rechtfertigungs- und Reziprozitätsdruck dem Sprachgebrauch in gewisser Hinsicht inhärent sind, ermöglicht und befördert jedoch nicht jede Art der Kommunikation Präferenztransformation im selben Maße. Stattdessen gibt es kontextuelle Parameter, von denen abhängt, ob Gründe herausgefordert, geprüft und überdacht werden, und die damit die Wahrscheinlichkeit einer Transformation der kognitiven und volitiven Prämissen von Präferenzen beeinflussen. Solche Kontextfaktoren sind entscheidend für unterschiedliche Interaktionsmodi, die mehr oder weniger vorteilhaft für Präferenztransformation sind.

8 Vgl. Herbert Paul Grice, Logik und Konversation, in: George Meggle (Hrsg.), Handlung, Kommunikation, Bedeutung, Frankfurt am Main 1979, S. 243 - 265; Robert B. Brandom, Making it Explicit. Reasoning, Representing, and Discursive Commitment, Cambridge 1994.

9

Jürgen Habermas, a.a.O. (Fn. 7), S. 287. 
Zwei Eigenschaften kommunikativer Interaktion scheinen im Kontext politischer Entscheidungsfindung zentrale Determinanten für die Wahrscheinlichkeit zu sein, mit der Präferenzwandel stattfindet: Diskursivität und Koordinativität.

\subsection{Diskursivität}

Die „Diskursivität“ von Interaktion ermöglicht und befördert den Austausch und die Prüfung von Gründen. Kommunikative Interaktion soll als diskursiv gelten, wenn sie zugleich öffentlich und dialogisch ist. Öffentlichkeit ist wichtig, um die Allgemeinheit und Übertragbarkeit von Gründen sicherzustellen. Es muss sich dabei aber keineswegs um massenmediale Öffentlichkeit handeln; worauf es ankommt, ist eine Logik der Öffentlichkeit: dass Interaktion prinzipiell für Außenstehende zugänglich ist, und das, was gesagt wird, von allen gehört werden darf und soll. Wie Jon Elster ${ }^{10}$ und Simone Chambers ${ }^{11}$ gezeigt haben, gibt es in der Regel Trade-Offs zwischen Öffentlichkeit und Qualität von Diskursen, so dass wir häufig mit der Entscheidung zwischen den zweitbesten Optionen des öffentlichen, aber rhetorischen (und nicht-dialogischen) Argumentierens und des nicht-öffentlichen, aber dialogischen Verhandelns konfrontiert sind. In der Tat scheint die Realisierung von Interaktionen schwierig, die zugleich öffentlich und dialogisch sind. Wenn die adressierte Zuhörerschaft jedoch nur im Prinzip, nicht aber notwendigerweise auch empirisch groß sein muss, damit Interaktion als öffentlich gelten kann, dann muss Diskursivität in unserem Sinne auch in der Realität keine Ausnahme sein.

In Bezug auf die dialogischen Qualitäten von Interaktion ist es wichtig, dass Behauptungen herausgefordert werden können und werden und dass jeder Hörer die Möglichkeit hat, selbst zum Sprecher zu werden. Habermas hat eine Unterscheidung zwischen dem Zuhörer und dem Hörer getroffen, derzufolge der Zuhörer auf eine passive Rolle beschränkt ist, in der er sich eine Meinung bilden und über die Behauptungen des Sprechers „Buch führen“ kann, aber nicht selbst Verpflichtungen eingehen, Fragen stellen oder die Behauptungen des Sprechers in Frage stellen kann. ${ }^{12}$ Der Hörer muss im Gegensatz hierzu zum Gesagten Stellung beziehen: Wenn er die Behauptungen eines Sprechers nicht explizit bestreitet, so weist er ihnen implizit Berechtigung zu und akzeptiert sie als Prämissen für weitere Überlegungen und Entscheidungen. ${ }^{13}$ Wenn Teilnehmer Hörer statt nur Zuhörer sind, dann sind sie auch an Interaktionsergebnisse gebunden, sofern sie diese nicht explizit in Frage gestellt haben.

10 Vgl. Jon Elster, Strategic Uses of Argument, in: Kenneth Arrow (Hrsg.), Barriers to Conflict Resolution, New York 1995, S. 236 - 257; Jon Elster, Deliberation and Constitution Making, in: ders. (Hrsg.), Deliberative Democracy, Cambridge 1998, S. 97 - 122.

11 Vgl. Simone Chambers, Behind Closed Doors: Publicity, Secrecy, and the Quality of Deliberation, in: Journal of Political Philosophy, 12. Jg. (2004), H. 4, S. 389 - 410; Simone Chambers, Measuring Publicity's Effect: Reconciling Empirical Research and Normative Theory, in: Acta Politica, 40. Jg. (2005), H. 2, S. $255-266$.

12 Zur Idee der diskursiven „Buchführung“ (score-keeping) über „,commitments“ (Verpflichtungen) und „entitlements“ (Berechtigungen) zu Behauptungen und Begründungen, siehe Robert B. Brandom, a.a.O. (Fn. 8).

13 Vgl. Jürgen Habermas, From Kant to Hegel: On Robert Brandom's Pragmatic Philosophy of Language, in: European Journal of Philosophy, 8. Jg. (2000), H. 3, S. $322-355$. 
Obgleich dieses Verständnis von Diskursivität offensichtlich von Habermas inspiriert ist, ist es zugleich sehr viel weniger anspruchsvoll als sein eigenes: Es beinhaltet keine Anforderung, derzufolge die institutionelle Konfiguration inklusiv und machtfrei sein soll und die Gesprächsteilnehmer müssen nicht notwendigerweise aufrichtig sein. Zugleich ist der hier verwendete Begriff vom „Diskurs“ sehr viel enger als die gebräuchliche und sehr weite Verwendung. Insbesondere wo er sich auf Michel Foucault bezieht, beinhaltet der Diskursbegriff in der Regel nicht allein Kommunikation, sondern auch kulturelle Praxis im weiteren Sinne und damit sehr viel mehr als der hier verwendete Begriff.

\subsection{Koordinativität}

Mit „Koordinativität“ von Interaktion ist gemeint, dass ein gewisser Druck besteht, in einer Situation zu einer Einigung zu gelangen, um eine kollektive Entscheidung treffen zu können und auf diese Weise individuelle Handlungspläne zu koordinieren. Ein solcher Druck kann extern begründet sein, etwa durch formelle Regeln und die Aufgaben eines kollektiven politischen Akteurs, aber er kann auch intern, aus den Erwartungen und Bestrebungen der Teilnehmer, sich zu einigen und ein gemeinsames Ergebnis zu präsentieren, entstehen.

Ein wichtiger Hinweis auf externen Druck ist die Entscheidungsregel des Forums, in dem Interaktion stattfindet, beziehungsweise die Art und Weise, in der mit Einigung und Nicht-Einigung umgegangen wird. Wenn gar keine Regel spezifiziert ist, werden Entscheidungen durch den Mangel an Koordinationsanreizen tendenziell schwierig. Eine Mehrheitsregel motiviert Koalitionsbildungen, wenn auch nicht über ideologische Grenzen oder konfligierende Interessen hinweg. Wo Konsens ein explizites Ziel ist oder Einstimmigkeit erzwungen wird, werden Kompromisse notwendig und die Koordinativität ist höher. Allerdings können Teilnehmer in einem kommunikativen Forum auch dort eine Einigung anstreben, wo Entscheidungszwang und explizite Entscheidungsregeln fehlen, und sich als Gruppe intern das Ziel setzen, zu einer gemeinsamen Position zu gelangen.

Wenn man die Faktoren Diskursivität und Koordinativität kombiniert ergibt sich die folgende Matrix politischer Interaktionsmodi (vgl. Schaubild 1). ${ }^{14}$ Jede der Zellen bestimmt idealtypisch einen Interaktionsmodus. Auch wenn die meisten realen Interaktionen irgendwo zwischen diesen Idealtypen zu verorten sein werden, sind diese von beträchtlichem Wert als Referenzpunkte für den Vergleich.

\begin{tabular}{|l|l|l|}
\hline Schaubild 1: Idealtypische Interaktionsmodi \\
\hline & \multicolumn{1}{|c|}{ nicht-koordinativ } & \multicolumn{1}{c|}{ koordinativ } \\
\hline diskursiv & Diskussion & Deliberation \\
\hline nicht-diskursiv & Debatte & Verhandeln \\
\hline Quelle: Eigene Darstellung. & \multicolumn{2}{|l}{} \\
\hline
\end{tabular}

14 Vgl. Claudia Landwehr, Deciding How to Decide. The Case of Health Care Rationing, in: Public Administration, 87. Jg. (2009), H. 3, S. $586-603$. 


\subsection{Typologie der Interaktionsmodi}

Die Bezeichnungen für die Idealtypen - Diskussion, Deliberation, Debatte und Verhandlung - sind in der Alltagssprache ebenso gebräuchlich wie in der Politikwissenschaft. Es ist daher wichtig, darauf hinzuweisen, dass es sich hierbei um spezifische Definitionen handelt, die zwar weitgehend mit dem üblichen Gebrauch übereinstimmen ${ }^{15}$, sich aber von den Definitionen anderer Autoren ${ }^{16}$ unterscheiden können.

Deliberation: Deliberation ist als der einzige Interaktionsmodus definiert, der zugleich diskursiv und koordinativ ist. Die Encyclopaedia Britannica beschreibt Deliberation als „a discussion and consideration by a group of persons of the reasons for and against a measure" (Hervorhebung hinzugefügt). Diese Beschreibung kommt der hier verwendeten Definition sehr nahe, ebenso wie der lateinische Ursprung „deliberare“: „gewichten, bedenken, reflektieren“. Im Vergleich zu einigen Explikationen des Begriffs in der umfassenden Literatur zur Theorie deliberativer Demokratie ist diese Definition eher sparsam, widerspricht der anspruchsvolleren Verwendungsweise von Habermas und anderen ${ }^{17}$ jedoch nicht. Hier soll von Deliberation die Rede sein, wenn Interaktion sowohl öffentlich als auch dialogisch, also diskursiv ist, und wenn sie von starken Koordinationsanreizen gekennzeichnet ist. Diese Koordinationsanreize können auf externem Druck beruhen, zum Beispiel, wenn das Forum eine Entscheidung einstimmig treffen muss. Sie können aber eben auch auf internen Bestrebungen beruhen, die etwa dann entstehen, wenn die Teilnehmer sich als Mitglieder einer Gruppe fühlen, in der Rolle von Bürgern oder Repräsentanten handeln und ein Gefühl der Verantwortung, zu einer gemeinsamen Entscheidung zu gelangen, entwickeln. Deliberation kann Kompromisse zwischen fundamentalen Werten und eine Konvergenz von Präferenzen ermöglichen. Sie ist daher der Interaktionsmodus, der für Präferenztransformation am förderlichsten ist.

Diskussion: Die Diskussion als Interaktionsmodus ist hier als diskursiv, aber nicht-koordinativ definiert. Die Encyclopaedia Britannica erklärt die Diskussion als „the consideration of a question in open and usually informal debate" (Hervorhebung hinzugefügt). Der lateinische Ursprung des Wortes verweist auf aggressivere Formen antagonistischer Interaktion: „discutio“ bedeutet „1. zerschlagen, 2. abschütteln, 3. prüfen, befragen“. Letztere Bedeutung betont Rechtfertigungsdruck und Diskursivität, die auch für die Definition hier zentral sind, derzufolge die Diskussion idealtypisch sowohl öffentlich als auch dialogisch ist. In politischen Foren ist die Rolle von Teilnehmern an einer Diskussion üblicherweise als die von „Experten“ bestimmt, und das Hauptziel der Interaktion ist die Information. In Diskussionen gibt es üblicherweise keine explizite Entscheidungsregel: Wenn Experten nicht durch Kommunikation allein zu einem Konsens gelangen (was eine häufige, aber

15 Wenn wir im Folgenden auf Lexikonbedeutungen der von uns benutzten Begriffe verweisen, bedeutet das nicht, dass wir Begriffsrealisten wären. Die Begriffe werden für unsere Typologie definiert. Wir versuchen uns lediglich an die wissenschaftstheoretische Norm zu halten, dass theoretische Terme möglichst nahe an ihrer natursprachlichen Bedeutung liegen sollten.

16 Vgl. Thomas Saretzki, Wie unterscheiden sich Argumentieren und Verhandeln? Definitionsprobleme, funktionale Bezüge und strukturelle Differenzen von zwei verschiedenen Kommunikationsmodi, in: Volker von Prittwitz (Hrsg.), Verhandeln und Argumentieren. Dialog, Interessen und Macht in der Umweltpolitik, Opladen 1996, S. 19 - 39.

17 Vgl. Jürg Steiner / Andre Bächtiger / Markus Spörndli / Marco R. Steenbergen, Deliberative Politics in Action, Cambridge 2005. 
kaum jemals erfüllte Erwartung ist), dann sollten sie kein materielles Interesse daran haben, durch Kompromisse Einstimmigkeit herzustellen. Obwohl die Diskussion die Begründung verbessern und das „Poolen“ von Informationen ermöglichen kann, ist Präferenzwandel ein eher unwahrscheinliches Ergebnis dieser Interaktionsform: Das Fehlen von Koordinationsanreizen verhindert notwendige Kompromisse zwischen praktischen Gründen, und der „Experte“ muss einen Gesichtsverlust befürchten, wenn er seine Position revidiert.

Verhandeln: Das Verhandeln ist als nicht-diskursiv definiert; obwohl dialogisch, ist es in seiner Logik nicht-öffentlich. Zugleich stellt es einen koordinativen Interaktionsmodus dar. Verhandlungen finden in Konstellationen statt, die die Möglichkeit von Kooperationsgewinnen implizieren. Es bestehen daher starke interne Anreize, eine Einigung zu finden, die allen Parteien nützt; wenn keine Einigung auf ein gemeinsames Vorgehen erzielt werden kann, können die Teilnehmer ihren Nutzen gegenüber dem Status quo nicht erhöhen. Folglich dürfen starke materielle Interessen an Kompromissen und Koordination vorausgesetzt werden. Die Definition der Encyclopaedia Britannica für „bargaining“ ist diesem Verständnis von Verhandeln sehr ähnlich: „1. to negotiate over the terms of a purchase, agreement or contract: haggle; 2. to come to terms: agree“. Die zweite Bedeutung bezieht sich klar auf den koordinativen Charakter der Interaktion. Auch wenn Verhandlungen auf Kompromisse zielen, befördert der idealtypische Mangel an diskursiven Interaktionsqualitäten nicht unbedingt genuine Präferenzveränderungen. Da kein Druck besteht, die eigenen Präferenzen mit Verweis auf Gründe zu rechtfertigen, werden diese auch nicht in Frage gestellt, geprüft und überdacht werden. Stattdessen versuchen Teilnehmer, ihre eigenen Präferenzen unter den Restriktionen, die die Präferenzen anderer Teilnehmer darstellen, zu maximieren. Das wahrscheinlichste Ergebnis einer Verhandlung ist, dass Akteure nach der Interaktion dieselben Präferenzen haben wie vorher, auch wenn sie sich möglicherweise auf eine gemeinsame Vorgehensweise geeinigt haben. Koordination ist erwünscht, und Kompromisse sind notwendig, aber Präferenzen müssen sich nicht ändern. Idealtypische Verhandlungskontexte sind in politischen Entscheidungsprozessen jedoch rar, da diese sich fast notwendig auf ethische Fragen und Präferenzen beziehen, die entsprechend gerechtfertigt werden müssen.

Debatte: Die Debatte ist als zugleich nicht-koordinativ und nicht-diskursiv definiert. Sie ist nicht-diskursiv, weil sie, obwohl öffentlich, nicht dialogisch ist: Es handelt sich hier eher um eine Sequenz von Monologen als um einen Dialog, und Behauptungen können nicht unmittelbar in Frage gestellt und zurückgewiesen werden. Folglich sind Zuhörer nicht auf die Ergebnisse der Interaktion verpflichtet. Die Encyclopaedia Britannica definiert die Debatte als "the formal, oral confrontation between two individuals, teams or groups who present arguments to support opposing sides of a question, generally according to a set form or procedure“ (Hervorhebung hinzugefügt). Der französische Ursprung in „débattre“ „besiegen, niederschlagen“ - verweist figurativ auf das Ziel der Teilnehmer in einer Debatte. Gruppen (mehr als eine!) sind im Voraus definiert, und sie präsentieren Argumente statt sie auszutauschen. Beispiele für Institutionalisierungen der Debatte sind Debattierclubs, Fernseh-Talkshows mit Politikern aller Parteien und insbesondere entscheidungsorientierte Plenardebatten in mehrheitsdemokratischen parlamentarischen Systemen. Während die Plenardebatte in einem Parlament wie dem Deutschen Bundestag Koordinationsbemühungen und Kompromisse innerhalb von Fraktionen und von Ausschüssen voraussetzt, ermöglicht die entscheidungsorientierte Debatte selbst keine weitere Koordination. Stattdessen dient sie der Mehrheit dazu, eine bereits getroffene Entscheidung zu verteidigen, und der 
Minderheit, diese anzugreifen. ${ }^{18}$ Auch wenn eine öffentliche Debatte, ob im Parlament oder in den Medien, die Präferenzen Dritter (der Zuhörer) beeinflussen mag, werden Präferenzänderungen der beteiligten Sprecher als Niederlage in einer Auseinandersetzung gesehen. Hieraus folgt, dass die Debatte als Interaktionsmodus für Präferenztransformation eher hinderlich als förderlich ist.

Jeder der idealtypischen Interaktionsmodi kann auf unterschiedliche Weise institutionalisiert werden. Für die Diskussion, die Debatte und das Verhandeln bestehen in den meisten politischen Systemen etablierte, wenn auch sehr unterschiedliche Foren: Diskussionen finden in Expertenkommissionen, Debatten in den Medien und im Plenum, Verhandlungen in parlamentarischen Ausschüssen und einer Vielzahl informeller Foren statt. Der am schwierigsten zu institutionalisierende Modus - die Deliberation - ist zugleich derjenige, der für Präferenztransformation am förderlichsten ist. Dies bedeutet jedoch nicht, dass sie auch der normativ wünschenswerteste Modus ist und dass die anderen Formen durch sie zu ersetzen wären. Vielmehr spielen alle vier Modi in demokratischen Entscheidungen essentielle Rollen. Der Beschluss über den Import embryonaler Stammzellen in Deutschland, als ein Beispiel eines multi-dimensionalen Konfliktes, der in unterschiedlichen Typen von Foren behandelt wurde, illustriert dies.

\section{Die Entscheidung über den Import embryonaler Stammzellen}

Im August 2000 schlug Oliver Brüstle, Neurobiologe an der Universität Bonn, der Deutschen Forschungsgemeinschaft ein Projekt vor, das die Arbeit mit importierten embryonalen Stammzellen (ES) vorsah. Es zeigte sich schnell, dass die bestehenden Gesetze auf diese durch den wissenschaftlichen Fortschritt hervorgerufene spezielle Herausforderung unzulänglich passten. Die Optionen, die die Fortschritte in der embryonalen Stammzellenforschung, insbesondere James Thompsons Erfolg in der Kultivierung menschlicher embryonaler Stammzellen im Jahr 1998, nun eröffneten, waren 1991 bei der Verabschiedung des ersten Gesetzes zum Schutz von Embryonen noch nicht absehbar gewesen. Dieses stellte zwar die Herstellung und Tötung von Embryonen für wissenschaftliche Zwecke unter Strafe, beinhaltete jedoch keine Richtlinien bezüglich des Imports embryonaler Stammzellen. Einerseits sind solche Zellen keine Embryonen, andererseits führte die Isolation dieser Stammzellen bisher unweigerlich zum Tod eines Embryos. Insofern könnte der Import von ES-Zellen die Anwendung von in Deutschland illegalen Methoden befördern und deutsche Wissenschaftler in eine gesetzliche Grauzone manövrieren. So einig sich politische Entscheidungsträger bezüglich des Handlungsbedarfs in dieser Angelegenheit waren, so fundamental war der entstehende Konflikt darüber, wie die neu entstandenen biomedizinischen Forschungsmöglichkeiten eingegrenzt werden konnten und sollten.

Oliver Brüstles Antrag auf Finanzierung eines Forschungsprojekts mit importierten ESZellen fachte in den Medien eine lebhafte Debatte an und machte die Notwendigkeit weitergehender Regulierung deutlich. Am 24. März 2001 setzte der Bundestag die Enquete-

18 Vgl. das Denkmodell „Debatte zur Erklärung der Entscheidung“ bei Wolfgang Zeh, Theorie und Praxis der Parlamentsdebatte, in: Hans-Peter Schneider / ders. (Hrsg.), Parlamentsrecht und Parlamentspraxis in der Bundesrepublik Deutschland. Ein Handbuch, Berlin 1989, \$ 32, S. 917 -937, S. 923. 
kommission „Recht und Ethik in der modernen Medizin“ ein, die aus 13 Abgeordneten und 13 Experten bestand. Einen Monat darauf wurde eine zweite Expertenkommission, der „Nationale Ethikrat“, einberufen, dem von der Bundesregierung ernannte Experten sowie einzelne Repräsentanten von Interessengruppen angehörten. Im Gegensatz zur Enquetekommission unterstand der Nationale Ethikrat also unmittelbar der Regierung. Sein erster Auftrag bestand im Entwurf einer Stellungnahme zur Frage des Imports embryonaler Stammzellen.

Am 5. Juli 2001 wurde der Stammzellimport erstmals im Bundestag verhandelt. Mit der Regierungsmehrheit nahm der Bundestag einen Antrag der Grünen und der SPD an, der eine ,gewissenhafte und umfassende Beurteilung des Imports und der Forschung mit embryonalen Stammzellen“ forderte, bevor sich der Bundestag noch im selben Jahr erneut mit dem Thema befassen würde. ${ }^{19}$ Im November 2001 präsentierten die beiden Expertengruppen ihre Ergebnisse - keines der Gremien war zu einem Konsens gelangt, und die Stellungnahmen skizzierten jeweils mehrere unterschiedliche Handlungsoptionen.

Während der Herbst- und Wintermonate entwickelte eine interfraktionelle Gruppe von Abgeordneten unter der Leitung von Maria Böhmer (CDU), Wolf-Michael Catenhusen (SPD), Andrea Fischer (Grüne) und Margot von Renesse (SPD) den schließlich erfolgreichen Kompromissantrag. Eine weitere interfraktionelle Gruppe erarbeitete einen Antrag für ein vollständiges Importverbot, während eine dritte den Import uneingeschränkt freigeben wollte. Die drei Anträge wurden am 30. Januar 2002 im Plenum des Bundestags diskutiert. ${ }^{20}$ Die Debatte wurde als eine Sternstunde des Parlamentes gefeiert, die Qualität und Atmosphäre der Verhandlung gepriesen. Die hier gewählte Vorgehensweise unterschied sich vom üblichen Gesetzgebungsverfahren durch die Aussetzung der Fraktionsdisziplin, was die Entscheidung für den einzelnen Abgeordneten zur „Gewissensentscheidung“ machte. Abgestimmt wurde in zwei Wahlgängen: zuerst über alle drei Anträge, dann zwischen dem Kompromiss- und dem Verbotsantrag, die im ersten Durchgang die meisten Stimmen erhalten hatten. Dabei wurde der Kompromissantrag 14/8102 angenommen.

Anschließend verfasste die Gruppe um Maria Böhmer in einer Reihe informeller Treffen einen Gesetzentwurf. Im Vergleich zu dem am 30. Januar vom Bundestag verabschiedeten Antrag spezifizierte der Entwurf mehrere Punkte, zu denen vorher nur vage Angaben gemacht worden waren. Die Formulierung des Gesetzentwurfs auf der Basis eines in einzelnen Punkten noch recht ungenauen Antrags wurde zumindest teilweise erst durch Aushandlungsprozesse zwischen entgegengesetzten Gruppen und Interessen möglich. Am 25. April 2002 wurde das Gesetz zur Sicherstellung des Embryonenschutzes im Zusammenhang mit der Einfuhr und Verwendung menschlicher embryonaler Stammzellen (Stammzellgesetz) vom Bundestag verabschiedet.

Im Jahr 2003 organisierte eine interdisziplinäre Arbeitsgruppe des Max-Delbrück-Zentrums für Molekularmedizin und des Forschungszentrums Jülich eine Bürgerkonferenz zur Stammzellforschung. Inspiriert vom dänischen Modell der Konsensuskonferenzen hofften die Initiatoren, die Debatte mithilfe von Standpunkten aus der „Lebenswelt“ der Bürger zu bereichern und die Vielfalt verschiedener Meinungen zum Thema abzubilden. Das erste Treffen dieser Bürgerkonferenz fand im Dezember 2003 statt, ein zweites und drittes Tref-

19 BT-Drs. 14/6551. Der Antrag der oppositionellen CDU/CSU-Fraktion (BT-Drs. 14/6314) forderte im Grunde das Gleiche, aber nannte für ein Moratorium andere Gründe.

20 BT-Drs. 14/8102 (Kompromiss), 14/8101 (Verbot) und 14/8103 (Freigabe). 
fen im Januar und März 2004. Im März 2004 wurde das Bürgervotum im Rahmen einer Pressekonferenz dem Bundestagspräsidenten übergeben. Obwohl die Bürgerkonferenz erst nach der Verabschiedung des Stammzellgesetzes stattfand, ist sie als Analysegegenstand für den Vergleich unterschiedlicher Interaktionsmodi und Foren der Entscheidungsbildung von Interesse.

Am 11. April 2008 verabschiedete der Bundestag, diesmal ohne nennenswerte öffentliche Debatte, eine Revision des Stammzellgesetzes, die unter anderem den Stichtag für die Herstellung von Stammzelllinien vom 1. Januar 2002 auf den 1. Mai 2007 verschob. Während die Verfechter der Neuregelung diese als Fortschreibung des Stammzellkompromisses sehen, sind dessen „Mütter“ der Ansicht, dass die Logik an dieser Stelle zerbricht. ${ }^{21}$ Die aktuelle Revision des Gesetzes ist jedoch nicht Gegenstand der vorliegenden Studie - diese beschränkt sich auf die „erste Runde“ des Stammzellkonfliktes.

Zurückkehrend zu den oben eingeführten idealtypischen Interaktionsmodi, scheint jeder Modus in mindestens einem der Foren, die an der ursprünglichen Entscheidung über den Import embryonaler Stammzellen beteiligt waren, institutionalisiert worden zu sein (vgl. Schaubild 2).

\begin{tabular}{|l|c|c|}
\hline Schaubild 2: Foren in der Stammzelldebatte \\
\hline & nicht-koordinativ & koordinativ \\
\hline \multirow{3}{*}{ diskursiv } & Diskussion: & Deliberation: \\
& Enquete-Kommission, & Bürgerkonferenz Stammzellforschung, \\
& Nationaler Ethikrat & Berlin 2003 \\
\hline \multirow{2}{*}{ nicht-diskursiv } & Debatte: & Verhandeln: \\
& Bundestagsdebatte & Ausarbeitung des \\
& 30. Januar 2002 & Gesetzentwurfes \\
\hline Quelle: Eigene Darstellung.
\end{tabular}

\section{Die Messung von Interaktionsmodi und Präferenzwandel}

Obwohl die Theorie deliberativer Demokratie von Autoren wie Habermas, Dryzek, Bohman oder Gutmann und Thompson ${ }^{22}$ zunächst als normative Theorie demokratischer Legitimität entwickelt wurde, werden ihre empirischen Annahmen zunehmend als Hypothesen untersucht. ${ }^{23}$ In der Tat scheint die Zahl der empirischen Forschungsarbeiten in diesem Bereich mittlerweile größer als die der normativ-theoretischen, was zu einer zunehmenden Diversität und Heterogenität von Ansätzen führt. ${ }^{24}$ Wie $M u t z$ feststellt, gehen viele empirische Studien in diesem Bereich der Frage nach, ob politische Interaktion in einem spezifischen

21 Vgl. Maria Böhmer / Andrea Fischer / Margot von Renesse, Die Logik zerbricht. Warum der Bundestag das Stammzellgesetz nicht ändern sollte, in: Die Zeit vom 10. April 2008, S. 13.

22 Vgl. Jürgen Habermas, a.a.O. (Fn. 7); John Dryzek, a.a.O. (Fn. 1); James Bohman, a.a.O. (Fn. 1); Amy Gutmann / Dennis Thompson, a.a.O. (Fn. 1).

23 Vgl. Diana C. Mutz, a.a.O. (Fn. 6); Dennis Thompson, a.a.O. (Fn. 5).

24 Vgl. Andre Bächtiger / Simon Niemeyer / Michael Neblo / Marco R. Steenbergen / Jürg Steiner, Disentangling Diversity in Deliberative Democracy, in: Journal of Political Philosophy, 18. Jg. (2010), im Erscheinen. 
Kontext Bedingungen genügt, als deliberativ zu gelten ${ }^{25}$ - so etwa der „Discourse Quality Index”, den die Gruppe um Jürg Steiner entwickelt hat ${ }^{26}$, oder Katharina Holzingers Verwendung der Sprechakttheorie, um Argumentations- von Verhandlungskontexten zu unterscheiden. ${ }^{27}$ Andere Forscher haben sich mit den Auswirkungen von Deliberation auf Meinungen, Präferenzen und Handlungspläne von Akteuren befasst. ${ }^{28}$

Mit der Analyse von Foren, die mit demselben politischen Konflikt befasst sind, geht es im vorliegenden Beitrag zugleich um den Prozess und das Ergebnis politischer Interaktion. Dies ist nur möglich, wenn man wichtige Aspekte der deliberativen Theorie, wie die Gleichheit der Teilnehmer, wechselseitigen Respekt, machtfreien Austausch von Argumenten oder die Qualität von Argumentationen, vernachlässigt. Damit ist die hier vorgenommene Messung von Präferenzwandel weit weniger komplex als die der oben zitierten Autoren (zum Teil aufgrund der betrachteten nicht-experimentellen Settings). Insbesondere verrät sie nichts darüber, ob die beobachteten Veränderungen wünschenswert sind oder nicht, ob also Präferenzen sich zum Guten oder zum Schlechten gewandelt haben. In diesem Sinne gewinnen die Ergebnisse ihre Relevanz zumindest teilweise erst in der Zusammenschau mit anderen Forschungsarbeiten.

Das Ziel der Analyse von Interaktion in den verschiedenen Foren, in denen der Stammzellenkonflikt erörtert wurde, besteht darin, die Wirkung unterschiedlicher Interaktionsmodi auf Akteurspräferenzen zu illustrieren und so eine erste Bestätigung oder Entkräftung der betreffenden Hypothesen zu gewinnen. Dementsprechend ergeben sich für die empirische Analyse folgende zentrale Fragen:

(1) In welchem Ausmaß war Interaktion diskursiv und koordinativ? Ermöglichen die jeweiligen institutionellen Kontextfaktoren Interaktion, die einem der Idealtypen nahe kommt?

(2) In welchem Ausmaß fand Präferenztransformation statt?

Die Analysemethoden wurden im Hinblick auf die zugrunde liegenden Forschungsfragen und die Art der verfügbaren Daten ausgewählt. Daten zu den institutionellen Eigenschaften eines Forums, beispielsweise zu seinem Aufbau, seinen Aufgaben oder Entscheidungsmechanismen, die als Indikator für externe Koordinativität dienen, sind verhältnismäßig leicht zu erfassen. Die Diskursivität und interne Koordinativität eines Forums sowie das Ausmaß und die Richtung der Präferenztransformation sind dagegen schwieriger messbar.

25 Vgl. Diana C. Mutz, a.a.O. (Fn. 6), S. 528.

26 Vgl. Marco R. Steenbergen / Andre Bächtiger / Markus Spörndli / Jürg Steiner, Measuring Political Deliberation. A Discourse Quality Index, in: Comparative European Politics, 1. Jg. (2003), S. 21 - 48; Andre Bächtiger, The Real World of Deliberation. A Comparative Study of its Favorable Conditions in Legislatures, Bern 2005; Jürg Steiner / Andre Bächtiger / Markus Spörndli / Marco R. Steenbergen, a.a.O. (Fn. 17).

27 Vgl. Katharina Holzinger, Verhandeln statt Argumentieren oder Verhandeln durch Argumentieren? Eine empirische Analyse auf der Basis der Sprechakttheorie, in: PVS, 42. Jg. (2001), H. 3, S. 414 - 446; dies., Bargaining Through Arguing: An Empirical Analysis Based on Speech Act Theory, in: Political Communication, 21. Jg. (2004), H. 2, S. 195 - 222; dies., Context or Conflict Types: Which Determines the Selection of Communication Mode, in: Acta Politica, 40. Jg. (2005), H. 2, S. $239-254$.

28 Vgl. James S. Fishkin, a.a.O. (Fn. 3); Simon Niemeyer, Deliberation in the Wilderness. Transforming Policy Preferences Through Discourse. Phd-Thesis, Australian National University 2002; Kasper M. Hansen, a.a.O. (Fn. 3); Erik Schneiderhan / Shamus Khan, Reasons and Inclusion. The Foundation of Deliberation, in: Sociological Theory, 26. Jg. (2008), H. 1, S. $1-24$. 
Im Idealfall sind Transkripte der Interaktion verfügbar und/oder direkte Observation möglich, um die Diskursivität und interne Koordinativität zu veranschlagen, zudem Interviews und/oder Fragebögen, um Präferenztransformation festzustellen.

Bedauerlicherweise waren für die Beantwortung der obenstehenden Fragen hinreichende Daten nur für zwei der vier Foren erhältlich: für die parlamentarische Debatte und die Bürgerkonferenz. Zum Glück sind dies auch die Fälle mit den eindeutigsten Vorhersagen zur Möglichkeit beziehungsweise Wahrscheinlichkeit von Präferenztransformation. Die Debatte schließt diese der Theorie nach aus, während die Bürgerkonferenz sie als Institutionalisierung von Deliberation stark begünstigt. Für diese Foren verfügen wir über Transkripte der Interaktion, Interviews und Beobachtungsberichte sowie über Abstimmungsdaten als „revealed preferences“. Zur Beurteilung der Diskursivität von Interaktion haben wir eine Sprechaktanalyse der Transkripte durchgeführt.

\subsection{Die Methode der Sprechaktanalyse}

Entwickelt von John Austin ${ }^{29}$ und John Searle $e^{30}$ im Kontext der linguistischen Pragmatik, fand die Sprechakttheorie mit Jürgen Habermas' „Theorie des kommunikativen Handelns“ Eingang in die Sozialwissenschaft ${ }^{31}$. Austin und Searle hatten aufgezeigt, dass ein Sprecher durch eine Äußerung im Gespräch eine Handlung ausführt, den Sprechakt. In einem politikwissenschaftlichen Kontext wurde die Methode der Sprechaktanalyse (SAA) erstmals verwendet, um eine empirische Basis für die Unterscheidung der Kommunikationsmodi „Argumentieren“ und „Verhandeln“ zu schaffen. ${ }^{32}$ Ähnliche Analysen finden sich bei Claudia Landwehr ${ }^{33}$ für eine parlamentarische Debatte und eine Bürgerkonferenz sowie bei Arzu Hatakoy ${ }^{34}$ für den europäischen Verfassungskonvent. Eine etwas andere Verwendung der Sprechaktanalyse zum besseren Verständnis der Herstellung kollektiver Verbindlichkeit in Gremien schlägt Frank Nullmeier vor. ${ }^{35}$

Der Fokus einer empirischen Analyse von Sprechakten, die in einer tatsächlichen Konversation vorgenommen werden, kann entweder auf dem Akt der Prädikation liegen, das heißt auf dem, was tatsächlich gesagt wurde und wie es gesagt wurde, auf der illokutiven Ebene, das heißt auf dem, was Akteure tun, indem sie sagen, was sie sagen, oder aber auf der perlokativen Ebene, das heißt auf der Wirkung von Sprechakten auf Gesprächspartner. Man betrachte folgendes Beispiel: „Wenn wir die globale Erwärmung nicht stoppen, wer-

29 John L. Austin, Zur Theorie der Sprechakte (How to do things with Words), Leipzig 2002 [1962].

$30 \mathrm{John}$ R. Searle, A Taxonomy of Illocutionary Acts. Expression and Meaning. Studies in the Theory of Speech Acts, Cambridge 1979, S. 1 - 39.

31 Jürgen Habermas, The Theory of Communicative Action, Boston 1984.

32 Vgl. Katharina Holzinger, Verhandeln statt Argumentieren, a.a.O. (Fn. 27); dies., Bargaining Through Arguing, a.a.O. (Fn. 27); dies., Context or Conflict Types, a.a.O. (Fn. 27).

33 Vgl. Claudia Landwehr, Political Conflict and Political Preferences. Communicative Interaction Between Facts, Norms and Interests, Colchester 2009.

34 Vgl. Arzu Hatakoy, The Effectiveness of Decision Making in European Union Treaty Negotiations, Dissertation an der FU Berlin, Berlin 2008.

35 Vgl. Frank Nullmeier, Sprechakttheorie und Textanalyse, in: Matthias Leonhard Maier / Achim Hurrelmann / ders. I Tanja Pritzlaff / Achim Wiesner (Hrsg.), Politik als Lernprozess? Wissenszentrierte Analysen der Politik, Opladen 2003, S. 211 - 223. 
den viele Arten aussterben. “ Auf der Ebene der Prädikation stellt dieser Satz eine kausale Beziehung zwischen der globalen Erwärmung und dem Aussterben von Arten her. Auf der illokutiven Ebene ist er, wenn er von einem Sprecher geäußert wird, eine Behauptung, oder präziser: eine Warnung. Auf der perlokutiven Ebene könnte die Warnung den Hörer dazu verleiten, an die kausale Beziehung zu glauben und persönliche Konsequenzen zu ziehen. Die Form der Sprechaktanalyse, die hier verwendet wird, konzentriert sich auf die illokutive Ebene und stellt die Frage, was illokutive Akte über den Interaktionsmodus in einer bestimmten Situation aussagen. Schaubild 3 zeigt, welche Argumentations- und Verhandlungssprechakte in der Analyse unterschieden wurden. ${ }^{36}$

\begin{tabular}{|l|l|}
\hline \multicolumn{1}{|c|}{ Schaubild 3: Argumentations- und Verhandlungsprechakte } \\
\hline \multicolumn{1}{|c|}{ Argumentationssprechakte } & \multicolumn{1}{|c|}{ Verhandlungssprechakte } \\
\hline behaupten & fordern \\
feststellen & anbieten \\
vermuten & vorschlagen (einen Kompromiss) \\
fragen & werben (für ein Angebot) \\
berichten & zugestehen \\
schließen & versprechen \\
rechtfertigen / begründen & drohen \\
beurteilen & akzeptieren (ein Angebot) \\
zustimmen / akzeptieren (eine Behauptung) & zurückweisen (ein Angebot) \\
widersprechen & aufrechterhalten (eine Forderung) \\
einräumen & zugestehen \\
beharren & (Nicht-)Einigung feststellen \\
zurücknehmen (eine Behauptung) & \\
(Nicht-) Übereinstimmung feststellen & \\
\hline Quelle: Eigene Darstellung.
\end{tabular}

Das Verhandeln ist als Interaktionsmodus insofern nicht-öffentlich, als es erstens oft hinter verschlossenen Türen stattfindet, und zweitens die verwendeten Begründungen eher privat und nicht übertragbar sind. Verhandlungssprechakte, zum Beispiel Fordern, VerspreCHEN oder Drohen, zielen in der Regel eher auf private Ressourcen oder Interessen als auf generalisierbare Begründungen und Argumente. Das Vorhandensein von Verhandlungssprechakten deutet hinsichtlich der Gründe der Akteure auf Nicht-Öffentlichkeit hin. Ihr Fehlen kann dagegen ein Indikator für die Öffentlichkeit von Gründen und Argumenten sein, die eine der zwei Bedingungen für Diskursivität darstellt. Das Fehlen von Argumentationssprechakten ist jedoch in keinem der Interaktionsmodi zu erwarten. Selbst wo das Argumentieren mehr oder weniger zwecklos ist, also ohne Bedeutung für das Interaktionsergebnis, ist es trotzdem notwendig, um das Gespräch im Gange zu halten. Darüber hinaus kann durchaus strategisch argumentiert werden. ${ }^{37}$ Das Vorhandensein von argumentativen Sprechakten allein sagt also wenig über die Öffentlichkeit der Interaktion aus.

Die zweite Bedingung für Diskursivität, die dialogische statt monologische Qualität von Interaktion, kann durch eine weitere Unterscheidung zwischen dialogischen und nichtdialogischen Sprechakten untersucht werden. Verhandelnde Sprechakte sind stets dialo- 
gisch. Eine weitere Untergliederung innerhalb dieser Kategorie ist daher für die Beurteilung von Diskursivität nicht erforderlich. Argumentieren kann dagegen entweder monologisch oder dialogisch sein, was sich auf der Ebene der illokutiven Akte zeigt. So scheinen mehrere der in der obenstehenden Liste definierten Argumentationssprechakte intrinsisch dialogisch zu sein.

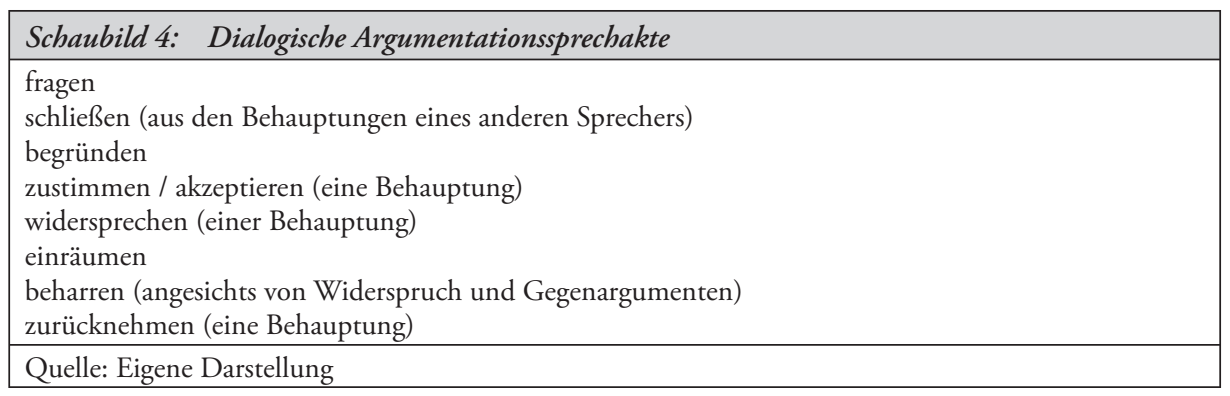

Nicht alle Sprechakte, die in natürlichen Gesprächssituationen vorkommen, sind in diesen Listen berücksichtigt. Weitere Kategorien sind rhetorische Sprechakte (zum Beispiel rhetorische Fragen, Zitate), diskursstrukturierende Elemente (zum Beispiel Begrüßungsformeln, Verweise auf andere Sprecher, Meta-Diskurse), expressive Sprechakte (die Gefühle ausdrücken sollen) und Deklarationen (zum Beispiel Eröffnung einer Sitzung). Einige davon kennzeichnen eine spezifische Form der Interaktion, zum Beispiel findet sich die rhetorische Sprache oft in öffentlichen Monologen. Um solche Unterschiede zwischen Interaktionen in den einzelnen Foren zu illustrieren, werden diese Kategorien mitgezählt, auch wenn die Beurteilung der Diskursivität lediglich auf dem Auftreten und dem Anteil von dialogischen argumentativen Sprechakten basiert.

Die Sprechaktanalyse untersucht linguistische Interaktion sehr detailliert. Ein Redebeitrag eines Sprechers besteht für gewöhnlich aus einer Vielzahl von Sprechakten. In den meisten Fällen stellt ein einzelner Satz, manchmal aber auch ein einzelnes Wort oder ein Ausdruck einen Sprechakt dar, in anderen Fällen besteht er aus mehreren Sätzen. In vorgefertigten Reden, wie sie zum Beispiel im Bundestag gehalten werden, sind einzelne Sprechakte meist leicht zu identifizieren. In spontanen Gesprächsbeiträgen dagegen beginnen die Sprecher oft einen Satz, zögern, korrigieren sich, paraphrasieren den Satz und wiederholen ihre Äußerung. In diesem Fall besteht ein Sprechakt aus mehreren Sätzen. Das folgende Beispiel eines Redebeitrages aus der Bundestagsdebatte, in dem den einzelnen Sätzen jeweils illokutive Akte zugeordnet wurden, illustriert die Methode:

„Dr. Ernst Ulrich von Weizsäcker (SPD): Herr Präsident! Frau Dahl! Meine sehr verehrten Damen und Herren! [BEgrüssen] Ich sehe alle drei Anträge als ethisch gut begründet und motiviert an. [Bewerten] Es ist niemanden, der sich einem der Anträge angeschlossen hat, vorzuwerfen, sich die Frage ethisch leicht gemacht oder sich gar aus niederen Motiven entschieden zu haben. [BeHaupten] Ich gestatte mir, insbesondere für den Antrag, der den Namen Margot von Renesse trägt, diese positive Einschätzung zum Ausdruck zu bringen. [BEWERTEN] Anfangs war ich durchaus in der Versuchung, mich dieser Initiative anzuschließen. [BerICHTEN] Doch was hat mich dazu gebracht, mich schließlich gegen eine Importerlaubnis auszusprechen? [FRAGEN, rhetorisch]“ 
Die für die Sprechaktanalyse der Debatte verwendeten Daten bestehen aus den Transkripten der 214. Sitzung des Bundestags am 30. Januar 2002, die etwa dreieinviertel Stunden dauerte. Aus den verfügbaren Transkripten der Bürgerkonferenz wurde eine Sitzung von vergleichbarer Dauer ausgewählt. ${ }^{38}$

In der Analyse der Bundestagsdebatte und der Bürgerkonferenz wurden für jeden Sprecher einzelne Sprechakte ausgezählt und typologisiert. Es mag eingewandt werden, dass die Isolation von Sprechakten und Einordnung in Kategorien wie Fordern, Berichten, FestSTELLEN oder VERMUTEN in nicht geringem Ausmaß subjektiv sei. Aus diesem Grund wurde die Kodierung von zwei unabhängigen Kodierern unter Zuhilfenahme eines Codebuchs vorgenommen, das die Definitionen der Sprechakte nach Kriterien von John Searle enthielt. Die Liste der Sprechakte war von den Kodierern nach einer ersten explorativen Analyse des Materials gemeinsam entwickelt worden. ${ }^{39}$ Für einzelne Gesprächsbeiträge fand sich zwischen den Kodierern eine Varianz von 10 bis 15 Prozent. Die Varianz betraf zumeist die Anzahl von Sprechakten eines bestimmten Typus, die innerhalb eines Einzelbeitrags gezählt wurden. ${ }^{40}$ In der Aggregation (das heißt in der Gesamtzahl der jeweiligen Sprechakttypen) hoben die Abweichungen sich meist gegenseitig auf, so dass es insgesamt keine systematische Verzerrung in eine bestimmte Richtung gegeben zu haben scheint. Insgesamt kann die Sprechaktanalyse also als hinreichend zuverlässiges Instrument betrachtet werden, das reproduzierbare Resultate hinsichtlich der Analyse von Interaktionsmodi liefert.

\subsection{Die Messung von Präferenzwandel}

Präferenzänderungen lassen sich entweder am Verhalten eines Akteurs ablesen oder aus dessen Selbstberichten in Befragungen oder Interviews. Bei der parlamentarischen Debatte erlaubte die namentliche Abstimmung Schlüsse auf der Grundlage des Abstimmungsverhaltens: Es wurde schlicht angenommen, dass bei den Abgeordneten, die für den Antrag stimmten, den sie vorher unterzeichnet hatten, kein Präferenzwandel vorlag. An die verbleibenden Abgeordneten, die keinen der drei Anträge unterzeichnet hatten, wurde ein kurzer Fragebogen versandt. Die wichtigsten Fragen hierbei waren, ob die Adressaten aus der Debatte neues Faktenwissen gewinnen konnten, ob sie neue Aspekte als relevant für die Bewertung erkannt und ob sie ihre Präferenzen zwischen den Anträgen während der Debatte verändert hatten oder von jemand anderem wussten, bei dem dies der Fall war.

38 In dieser Sitzung diskutieren die Bürger darüber, welche Aspekte des Themas die höchste Relevanz haben und welche Fragen sie den Experten in der letzten Anhörung stellen wollen. Sie wurde ausgewählt, weil sie keine Präsentationen von Experten beinhaltet und weil sie am zweiten Wochenende stattfand, an dem anfängliche Organisationsprobleme bereits gelöst waren. Die Interaktion schien deshalb dem idealtypischen Modus der Bürgerdeliberation am nächsten zu kommen.

39 Die Transkripte der parlamentarischen Debatte wurden seitens der Autoren unabhängig kodiert, die Transkripte der Bürgerkonferenz von [xx] und [yy]r. Alle Kodierer waren mit der Sprechaktanalyse vertraut.

40 Zum Beispiel neigte Kodierer 3 dazu, mehrere Fragen zu registrieren, wo Kodierer 1 nur eine registrierte, die ein- oder zweimal paraphrasiert worden war. Eine weitere in gewisser Weise systematische Varianz betraf die Klassifizierung von Sprechakten als Beurteilen (damit als Argumentationssprechakt) oder BEWERTEN (damit als Verhandlungssprechakt), wo Kodierer 3 häufiger ersteren identifizierte und Kodierer 1 häufiger letzteren. 
Für die Bürgerkonferenz ist eine umfassende Evaluation von Jörg Henning und Birgit Erdwien $^{41}$ verfügbar. Die gesamte Veranstaltung wurde mithilfe von Tonband- und Videoaufzeichnungen ausführlich dokumentiert. Vor der ersten und nach jeder darauf folgenden Sitzung füllten die Teilnehmer Fragebogen zur Atmosphäre in der Gruppe, zu ihrer Einschätzung des Verlaufs und zu ihren eigenen Gefühlen und Meinungen aus. Antworten auf Fragen, die die eigene Kenntnis der Thematik und die vergleichende Bewertung von Argumenten für und gegen die Forschung mit embryonalen Stammzellen betreffen, dienen als Indikatoren für Präferenzwandel. Weiterhin weist die qualitative Einordnung der Konferenz seitens der teilnehmenden Beobachter auf Präferenzwandel hin.

\section{Diskurs, Koordination und Akteurspräferenzen in Parlamentsplenum und Bürgerkonferenz}

\subsection{Diskursivität}

Die Bundestagsdebatte über den Import embryonaler Stammzellen am 30. Januar 2002 war vollständig öffentlich, in mancherlei Hinsicht sogar mehr als andere Debatten. Wenngleich alle Sitzungen des Bundestages öffentlich sind, genoss diese besondere Aufmerksamkeit, wurde im Fernsehen live übertragen und von einer Vielzahl von Journalisten analysiert. Sowohl für den einzelnen Sprecher als auch für die Institution als solche hing von dem dortigen Auftreten viel Reputation und Ansehen ab.

Trotz des hohen Niveaus der Argumentation kann die Bundestagsdebatte entsprechend unserer Definition nicht als diskursiv bezeichnet werden und entspricht damit dem idealtypischen Interaktionsmodus der Debatte. Wie die Ergebnisse der Sprechaktanalyse deutlich zeigen (siehe unten), erfüllt die Plenardebatte nicht das Kriterium der dialogischen Interaktion. Offenbar haben grundlegende Interaktionslogiken und Verfahrensanforderungen in dieser Konfiguration den Dialog und damit die Diskursivität verhindert. Die Redezeiten im Bundestag werden auf Basis der Anzahl der Unterzeichner eines Antrages zugeteilt, und die Liste der Redner wird im Vorhinein festgelegt. Dadurch findet eine Aufspaltung des Forums in Sprecher und Zuhörer statt, wobei es für die Zuhörer nahezu unmöglich ist, selbst Sprecher zu werden, ohne eine Störung zu verursachen. Die Redner haben ihre Beiträge im Voraus angefertigt, und die Rednerliste sowie begrenzte Sprechzeiten müssen strikt durchgesetzt werden.

In der Bürgerkonferenz bemühten sich die Organisatoren, Öffentlichkeit und Dialogqualität zugleich zu gewährleisten. Ein Teil der Konferenz, die Expertenanhörung, war vollständig öffentlich und wurde von vielen interessierten Bürgern besucht. Während der Plenumssitzungen stellten ein Publikum aus teilnehmenden Beobachtern und die Tonbandaufzeichnungen in gewissem Umfang Öffentlichkeit her. Die dialogischen Eigenschaften wurden durch eine verhältnismäßig kleine Forumsgröße ermöglicht und durch das ermutigende Auftreten der Moderatoren verstärkt. Die klare Intention dieser Verfahrensregeln und der Anordnung des Forums bestand darin, dass es jedem der Teilnehmer möglich sein sollte, jederzeit das Wort zu ergreifen.

41 Hans Jörg Henning / Birgitt Erdwien, Bürgerkonferenz zur Stammzellforschung 2003/2004, Abschlussbericht, Universität Bremen, Institut für Psychologie, Bremen 2004, S. 1 - 94. 
In der Mikroanalyse der einzelnen Sprechakte zeigen sich die idealtypischen Aspekte der Interaktion sogar noch deutlicher. Die folgende Tabelle illustriert die Häufigkeit der verschiedenen Typen von Sprechakten in der Debatte und der Deliberation.

\begin{tabular}{|l|r|r|c|c|}
\hline \multicolumn{5}{|c|}{ Tabelle 1: Typen von Sprechakten in Bundestagsdebatte und Bürgerkonferenz } \\
\hline \multicolumn{1}{|c|}{ Sprechakttyp } & Bundestag & Bürgerkonferenz \\
\hline & Anzahl & Prozent & Anzahl & Prozent \\
\hline Argumentation & 1061 & 76 & 636 & 70 \\
Verhandlung & 32 & 2 & 135 & 15 \\
rhetorisch & 41 & 3 & 1 & 0 \\
Diskurs-Strukturierer & 226 & 16 & 103 & 11 \\
Expressive & 36 & 3 & 25 & 3 \\
Andere & 0 & 0 & 8 & 1 \\
\hline * Beiträge von Moderatoren und Organisatoren sind hier nicht berücksichtigt. \\
Quelle: Eigene Daten.
\end{tabular}

In beiden Fällen schlägt sich der Effekt der Öffentlichkeit auf die Interaktion - auf der illokutiven Ebene - in der Dominanz argumentativer Sprechakte nieder. Bei genauerer Untersuchung des prädikativen Inhalts der Äußerungen (der hier nicht analysiert wird) zeigt sich, dass der Rückgriff auf nicht generalisierbare, private Gründe gänzlich vermieden wird. Argumentative Sprechakte machen drei Viertel der Gesamtzahl aus, wobei der Prozentsatz in der Debatte noch höher liegt. Um Verhandlungssprechakte handelt es sich hier nur in zwei Prozent der Fälle, in der Bürgerkonferenz dagegen in 15 Prozent. Die Öffentlichkeit und der Rechtfertigungscharakter der Debatte erklären die nicht unerhebliche Zahl von rein rhetorischen Sprechakten und Expressiven.

Zur Beurteilung des Dialogcharakters von Interaktion ist der Anteil der spezifisch dialogischen Sprechakte an den argumentativen Sprechakten von besonderer Bedeutung. Mit nur 16 Prozent machen diese kaum mehr als ein Fünftel der Gesamtmenge in der Debatte aus. Dass dieser Prozentsatz auffällig klein ist, wird im Vergleich mit den Ergebnissen der Bürgerkonferenz deutlich, wo mehr als die Hälfte der argumentativen Sprechakte (56 Prozent) auch dialogisch ist (siehe Tabelle 2).

\begin{tabular}{|l|c|c|c|c|}
\hline \multicolumn{1}{|c|}{ Tabelle 2: Argumentationssprechakte in Bundestag und Bürgerkonferenz } \\
\hline \multicolumn{1}{|c|}{ Sprechakttyp } & Anzahl & Prozent & Anzahl & Prozent \\
\hline & 169 & 16 & 359 & 56 \\
\hline dialogisch & 223 & 21 & 140 & 22 \\
feststellen & 500 & 47 & 48 & 8 \\
behaupten & 169 & 16 & 89 & 14 \\
andere & * Beiträge von Moderatoren und Organisatoren sind hier nicht berücksichtigt. \\
Quelle: Eigene Daten.
\end{tabular}


Die in der parlamentarischen Debatte dominanten Typen argumentativer Sprechakte sind eindeutig das Behaupten und das Feststellen. ${ }^{42}$ Viele Beiträge bestehen im Wesentlichen aus einer Aufzählung von Behauptungen. Dies wird durch die sehr spezifische Situation des öffentlichen Monologs ermöglicht. In dialogischer Interaktion werden solche Behauptungen oft angefochten, und es ist weit schwieriger, Annahmen als gegebene Tatsachen vorauszusetzen. Die Möglichkeit herausgefordert zu werden, hält die Redner eher von kontroversen Behauptungen ab, sofern sie nicht fähig und willens sind, diese zu verteidigen.

\begin{tabular}{|l|c|c|c|c|}
\hline \multicolumn{1}{|c|}{ Tabelle 3: Spezifizierung der Sprechakttypen in Bundestag und Bürgerkonferenz im Vergleich } \\
\hline \multicolumn{1}{|c|}{ Sprechakt } & Bundestag & Bürgerkonferenz \\
\hline & Anzahl & Prozent & Anzahl & Prozent \\
\hline fragen & 0 & 0 & 67 & 19 \\
\hline zurücknehmen & 0 & 0 & 1 & $<1$ \\
\hline beharren & 1 & $<1$ & 17 & 5 \\
\hline einräumen & 16 & 9 & 6 & 2 \\
\hline widersprechen & 25 & 15 & 66 & 18 \\
\hline zustimmen & 11 & 7 & 48 & 13 \\
\hline schließen & 52 & 31 & 22 & 6 \\
\hline begründen & 64 & 37 & 132 & 37 \\
\hline Summe & 169 & 369 & \\
\hline \multirow{2}{*}{$\begin{array}{l}\text { Beiträge von Moderatoren und Organisatoren sind hier nicht berücksichtigt. } \\
\text { Quelle: Eigene Daten. }\end{array}$}
\end{tabular}

Die nähere Betrachtung der Typen dialogischer Sprechakte, die in den beiden Foren jeweils am häufigsten vorkamen, ist ebenfalls sehr aufschlussreich (siehe Tabelle 3). In der parlamentarischen Debatte ist eine deutliche Dominanz von Folgerungen und Rechtfertigungen erkennbar. In der Bürgerkonferenz ist der Anteil der Rechtfertigungen ähnlich, wobei andere dialogische Sprechakte einen weit größeren Prozentsatz ausmachen. Dies kennzeichnet den Produktcharakter der Argumentation in der parlamentarischen Debatte im Gegensatz zu ihrem Prozesscharakter in der Bürgerkonferenz. Obgleich Folgerungen und Rechtfertigungen als dialogische Sprechakte betrachtet werden können und sollen, sind sie für das Produkt und den Prozess der Argumentation gleichermaßen wesentlich. Fragen, BeharREN, Widersprechen oder Zustimmen sind im Gegensatz hierzu eher kennzeichnend für den Prozess als für das Produkt: Sie geben an, in welchen Bereichen weitere Rechtfertigung nötig ist. Es sollte außerdem beachtet werden, dass die Gesamtzahl an Rechtfertigungen und Folgerungen in der Debatte weit niedriger liegt als in der Bürgerkonferenz. Dies scheint in Anbetracht des Rechtfertigungscharakters parlamentarischer Kommunikation verwunderlich.

42 Die Unterscheidung der beiden basiert auf der Annahme, dass es sich bei Behauptungen um Geltungsansprüche handelt, bei denen der Sprecher noch eine Kontroverse anerkennt, während Feststellungen eine Übereinkunft voraussetzen, also etwas als gegeben angenommen wird (zum Beispiel „Wie wir wissen ..."; „Wie bereits gezeigt ..."). 


\subsection{Koordinativität}

In parlamentarischen Regierungssystemen wie Deutschland, wo die Regierung immer mit einer sicheren Gewinnkoalition ausgestattet ist, erscheint Koordination im parlamentarischen Plenum weder möglich noch notwendig, da die notwendige Mehrheitskoalition bereits existiert und in der Alltagspraxis der wechselseitigen Kommunikation von Regierung und Mehrheit politisch-inhaltlich ständig aktualisiert wird. ${ }^{43}$ Im Vergleich mit üblichen Gesetzgebungsprozessen und Plenardebatten war die Befassung mit dem Import embryonaler Stammzellen sicherlich ein Ausnahmefall. Die Fraktionsdisziplin war aufgehoben, um den Abgeordneten zu ermöglichen, die Angelegenheit als Gewissensfrage zu betrachten. Da die Entscheidung anders als im Regelfall nicht von Experten innerhalb der Fraktionen vorgegeben war und daher von den Stimmen der Mehrheit biopolitischer Laien im Bundestag abhing, standen die Abgeordneten in ihrer Meinungs- und Präferenzbildung unter besonderem Druck und waren einer ungewöhnlichen öffentlichen Beobachtung ausgesetzt. Da die meisten von ihnen das Ergebnis als völlig offen betrachteten, nahmen sie die Aufgabe als besondere institutionelle Verantwortung wahr. So schien in der Tat eine „Sternstunde“ vonnöten, um eine verantwortungsvolle Entscheidung von solcher Komplexität und Konsequenz zu treffen. Die Debatte und die Entscheidung, so wurde unterstellt, würden auch etwas über die Fähigkeit des Parlaments aussagen, mit zukünftigen Herausforderungen ähnlicher Art umzugehen.

Die Stammzell-Debatte im Bundestag weicht somit in mehrfacher Hinsicht vom Idealtyp ab. Keiner der drei Anträge hatte eine absolute Mehrheit der Abgeordneten hinter sich versammeln können, und nur drei Viertel hatten überhaupt einen Antrag unterzeichnet. Um eine absolute Mehrheit zu erreichen, waren die Antragsführer auf zusätzliche Unterstützung angewiesen. In Anbetracht des nicht-dialogischen Modus der Interaktion bleibt jedoch zweifelhaft, ob selbst unter solch günstigen Umständen das für eine Mehrheit nötige Ausmaß an Koordination im Rahmen einer Plenardebatte möglich ist.

Die Sprecher für die zwei radikaleren Anträge - also vollständiges Verbot beziehungsweise vollständige Freigabe des Imports - waren sich der Attraktivität eines Mittelwegs besonders für unentschlossene oder weniger informierte Parlamentarier bewusst. Sie hofften, dieser Tendenz entgegenzuwirken, indem sie Maßstäbe von Konsistenz und logischer Konsequenz betonten. Die Befürworter des Kompromissantrages bemühten sich, die Semantik des Kompromisses und den Eindruck von Zugeständnissen an beide Seiten zu vermeiden. Stattdessen versuchten sie, eine Interpretation der erfolgreichen Verständigung durchzusetzen. Gegen das rhetorisch machtvolle Bild des Dammbruchs stellten sie Bilder des Brückenbaus, einer kurvenreichen Straße oder eines stark bewachten Tores. Sie sprachen auch die Qualität und Unabgeschlossenheit der Debatte an und drückten ihren besonderen Respekt für diejenigen aus, die negative soziale Konsequenzen befürchteten, wenn der Status des Embryos einmal angetastet würde. Das Motiv für diese Rechtfertigungsstrategie lag möglicherweise darin, zu zeigen, dass trotz der auf hohem argumentativen Niveau geführten Debatte Begründungen unvollständig blieben und ein Konsens außer Reichweite gerate, dass also eine politische Entscheidung unumgänglich sei: Weitergehende Kommuni-

43 In präsidentiellen Systemen ist die Institutionalisierung der Debatte als idealtypischer Interaktionsmodus im parlamentarischen Plenum weniger wahrscheinlich. Vielmehr ist hier fast immer eine Art von Koordination erforderlich. 
kation würde demzufolge nicht etwa die eine richtige Lösung enthüllen, sondern vielmehr die moralische Kluft vertiefen.

In dieser besonderen Debatte sahen sich die Sprecher vor einer doppelten Herausforderung: Einerseits wetteiferten sie um die Stimmen der noch immer unentschlossenen Kollegen, die keinen der Anträge unterzeichnet hatten. Andererseits, und trotz des Umstands, dass über Anträge und nicht über Gesetzesvorlagen abgestimmt wurde, mussten sie die parlamentarische Funktion erfüllen, ihre Entscheidung vor der Öffentlichkeit zu rechtfertigen. Beide Aufgaben machten Koordination, die Kompromisse über praktische Gründe erforderlich machen würde, schwierig.

Im Falle der Bürgerkonferenz kann von mindestens drei Aspekten angenommen werden, dass sie die Koordinativität im Vergleich zum Idealtyp der Deliberation deutlich reduziert haben. Der erste ist die Absicht des Organisationskomitees, die Vielfalt der Meinungen und Werte darzustellen, statt aktiv einen Konsens anzustreben. Dies zeigte sich auch im Verzicht auf das „Konsensus“-Etikett. Obwohl das Forum nach dem Vorbild der dänischen Konsenskonferenzen gestaltet worden war, zogen die Organisatoren den Begriff der „Bürgerkonferenz" vor, um die Implikation der Einstimmigkeit zu vermeiden, die ihrer Meinung nach die soziale Realität hinsichtlich des Themas Stammzellforschung nicht widerspiegelte. ${ }^{44}$ Ein zweiter die Koordinativität reduzierender Faktor war der Umstand, dass die Konferenz stattfand, als der Bundestag die Entscheidung bereits getroffen hatte, so dass das Bürgervotum keinen unmittelbaren Einfluss auf eine politische Entscheidung nehmen konnte. Schließlich hatten die Bürger auch kein privates, materielles Interesse an der Entscheidung, und es wurde ein besonderer Schwerpunkt auf die Repräsentativität der Bürgergruppe gelegt, die durch ein anspruchsvolles System der Zufallsauswahl gewährleistet wurde. Dies gab den Teilnehmern die Möglichkeit zu einem einfachen und mehr oder weniger kostenfreien Ausstieg aus dem Verfahren, was dessen Koordinationspotenzial weiter einschränkte. Als das Bürgervotum im März 2004 dem Bundestagspräsidenten überreicht wurde, hatten fünf von zwanzig Mitgliedern die Gruppe bereits verlassen. Insgesamt war der externe Koordinationsdruck in der Bürgerkonferenz also nicht sehr hoch.

Daher ist interessant, dass koordinative Verhandlungssprechakte im Deliberations-Setting überraschend häufig vorkommen (vgl. Tabelle 1). Die Öffentlichkeit war in der Bürgerkonferenz geringer als in der Debatte, was die Anreize für Verhandeln prinzipiell erhöht. In Anbetracht der Tatsache, dass die Teilnehmer kein materielles Interesse an den Ergebnissen des Verfahrens hatten und die Exit-Option ständig zur Verfügung stand, erscheint ein Verhandeln im klassischen distributiven Sinne für diese Konfiguration jedoch unwahrscheinlich. Darüber hinaus liegt der gesamte Prozentsatz der argumentativen Sprechakte in der Debatte nur marginal höher als in der Bürgerkonferenz, so dass das Verhandeln das Argumentieren nicht beeinträchtigt zu haben scheint. Bei genauer Betrachtung der Situationen, in denen Verhandlungssprechakte vorkommen, zeigt sich, dass diese hauptsächlich in einem Kontext prozessualen Meta-Diskurses auftreten. Verhandelt wird darüber, wann die Sitzung am folgenden Tag beginnen soll oder ob ein Thema in Kleingruppen oder im

44 Vgl. Ali ben Salem / Christof Tannert, Planung und Organisation der Bürgerkonferenz, in: Christof Tannert / Peter Wiedemann (Hrsg.), Stammzellen im Diskurs: Ein Lese- und Arbeitsbuch zu einer Bürgerkonferenz, München 2004, S. 106 - 116, S. 106. 
Plenum zu diskutieren ist. Die meisten der Verhandlungssprechakte sind Vorschläge oder Bitten, Drohungen oder Versprechen kommen nicht vor.

Die Transkripte zeigen hinsichtlich des notwendigen Grades an Koordination eine mehr oder weniger profunde Unsicherheit sowohl bei den Organisatoren als auch bei den Teilnehmern. Die Organisatoren und Moderatoren hatten ein klares (sogar materielles) Interesse am Erfolg der Konferenz. Erfolgskriterien sind die Einhaltung des Zeitplans, keine oder eine möglichst kleine Zahl an Austritten und vor allem ein vorzeigbares Ergebnis in Form des Bürgervotums. Gleichzeitig mussten sie jeden Eindruck autoritärer Einflussnahme auf das Verfahren und die Meinungen der Teilnehmer vermeiden. Auf der Seite der Bürger lenkte der starke Wunsch, zu lernen und alle relevanten Aspekte des Themas zu berücksichtigen, vom Ziel eines gemeinsamen (oder sogar einstimmigen) Votums ab.

In der Gruppe bestand jedoch eine große Sensibilität für diese Problematik, und die Teilnehmer hatten eindeutig höher gesteckte Erwartungen an die Konferenz als die Organisatoren. Statt sich mit der Darstellung der allgemein bekannten Meinungsvielfalt und des unauflösbaren moralischen Konfliktes zufrieden zu geben, betrachteten sie ihre Ergebnisse als wichtigen Input für den politischen Prozess. Darüber hinaus erkannten sie, dass nur eine klare Mehrheit oder ein Konsens Politiker veranlassen könnte, ihre Positionen zu überdenken. Sowohl in den Fragebögen als auch in den Diskussionen kritisierten die Teilnehmer, dass die Expertenanhörungen und deren Vorbereitung zu viel Zeit geraubt hätten, die besser für die Ausarbeitung des Votums hätte genutzt werden können - und somit für Koordination in der Gruppe. Insgesamt scheint es, als hätte die gruppeninterne Dynamik die für Koordination weniger günstigen Kontextfaktoren zumindest teilweise aufgewogen.

Wenn man das nicht-konsensuelle Votum betrachtet, erscheinen die koordinativen Qualitäten der Bürgerkonferenz zunächst eher gering. Es muss jedoch bedacht werden, dass in der Bürgergruppe nur geringe Vorkenntnisse zur embryonalen Stammzellforschung bestanden und dass die meisten Mitglieder sich mit der Fachterminologie erst vertraut machen mussten. Demzufolge wurde ein großer Anteil der Konferenzzeit der Information und der Vorbereitung der Expertenanhörung gewidmet. Angesichts des daraus resultierenden Zeitmangels ist der erreichte Grad an Koordination nicht unerheblich. Zudem ist nicht nur der konsensuelle Teil des Votums recht umfassend, sondern es konnte auch in Bereichen Einigung erzielt werden, die nur scheinbar reine Verfahrensangelegenheiten waren, etwa welche Experten eingeladen und welche Fragen gestellt werden sollten.

Wenn der Konsens von vornherein als Haupterfolgskriterium behandelt worden wäre und die Gruppe mehr Zeit für die Koordination gehabt hätte, wäre ein einvernehmliches Votum sicherlich eher möglich gewesen. Angesichts der bereits hohen Anzahl an Ausstiegen ist jedoch davon auszugehen, dass der Koordinationsprozess die Gruppengröße weiter reduziert hätte. Diese häufige Inanspruchnahme der Exit-Option von benachteiligten und unzufriedenen Mitgliedern mit Minderheitsmeinungen wirft einen weiteren Schatten auf die ohnehin eingeschränkte demokratische Legitimität von Konsenskonferenzen.

\subsection{Präferenztransformation}

Die der Bundestagsdebatte vom 30. Januar folgende Abstimmung war eine namentliche, zu der alle Abgeordneten im Plenum erscheinen und ihre Stimme abgeben mussten, die dann mit ihrem Namen und Wahlkreis registriert wurde. Mehr als 75 Prozent der Abgeordneten 
hatten einen der Anträge unterschrieben. Ein Grund, aus dem einige Parlamentarier weiterhin zögerten, sich einem der Anträge anzuschließen, ist darin zu suchen, dass sie trotz der ausgesetzten Fraktionsdisziplin nicht willens waren, sich gegen die Mehrheit der eigenen Fraktion auszusprechen. Allerdings gab es auch eine beträchtliche Anzahl unentschiedener oder schlecht informierter Mitglieder. Da die 138 Abgeordneten, die keinen der Anträge unterschrieben hatten, das Votum prinzipiell entweder in Richtung des Verbots oder des Kompromisses beeinflussen konnten, wurde das Ergebnis der Entscheidung als mehr oder weniger offen betrachtet. Dementsprechend - und im Gegensatz zu anderen Debatten schien Raum für die Präferenzformation und -transformation während der Debatte vorhanden zu sein. Zumindest hinsichtlich der so geäußerten Präferenzen erlaubt die namentliche Abstimmung also Aussagen über die Häufigkeit von Präferenzänderung im Plenum.

Ein eindeutiges Anzeichen für Präferenztransformation würde vorliegen, wenn Parlamentarier für einen anderen Antrag stimmen, als sie unterzeichnet haben. In diesem Fall hätte die Konfrontation mit neuen Informationen und Argumenten trotz der sonst ungünstigen Bedingungen offensichtlich Auswirkungen auf die Präferenzbildung gehabt. Doch widerlegt ein Blick auf die Ergebnisse der Abstimmung diese Möglichkeit schnell. Mit einer einzigen Ausnahme hat keines der 460 Mitglieder, die bereits einen Antrag unterzeichnet hatten, in der ersten Runde für einen anderen gestimmt. ${ }^{45}$ Dies überrascht wenig: Mit ihrer Unterschrift unter einen der Anträge zeigen die Parlamentarier, dass sie ihre Meinung im Bewusstsein der relevanten Argumente gebildet haben.

Folglich muss das Augenmerk auf diejenigen 138 Mitglieder des Bundestags gerichtet werden, die keinen der Anträge unterzeichnet hatten. Die Überzeugungsversuche der Redner waren in erster Linie an diese „unentschlossenen“ Mitglieder gerichtet. Tabelle 4 zeigt, dass die Anzahl derjenigen, die für das Verbot stimmten, unter den Antragsunterzeichnern

\begin{tabular}{|l|c|c|c|c|c|c|c|}
\hline \multicolumn{2}{|c|}{ Tabelle 4: Stimmverhalten von Unterzeichnern und Nicht-Unterzeichnern der Anträge } \\
\hline \multicolumn{1}{|c|}{ Mitglieder } & Stimmen (Summe) & \multicolumn{2}{c|}{ Unterzeichner } & Nichtunterzeichner & $\begin{array}{c}\text { Stimmen- } \\
\text { gewinn }\end{array}$ \\
\hline \multicolumn{1}{|c|}{ Antrag } & Anzahl & Prozent & Anzahl & Prozent & Anzahl & Prozent & Prozent \\
\hline Verbot & 263 & 42,62 & 217 & 47,17 & 46 & 33,33 & $-13,84$ \\
Kompromiss & 225 & 36,46 & 163 & 35,43 & 62 & 44,92 & $+9,49$ \\
Zulassung & 106 & 17,17 & 80 & 17,39 & 26 & 18,84 & $+1,45$ \\
Enthaltung & 4 & 0,66 & 0 & 0 & 4 & 2,89 & \\
\hline Summe & 598 & 100 & 460 & 100 & 138 & 100 & \\
\hline * Anteil der Stimmen, die jeder der Anträge im Vergleich zur Zahl seiner Unterzeichner in der Abstim- \\
mung hinzugewonnen hat. \\
Quelle: Eigene Berechnungen. \\
\hline
\end{tabular}

45 Dieser Abgeordnete hatte den Kompromissantrag unterzeichnet, stimmte aber im ersten Wahlgang für das Verbot. Im zweiten Wahlgang, der eine Stichwahl zwischen dem Verbots- und dem Kompromissantrag war, stimmte er für den Kompromiss. Mögliche Erklärungen sind, dass er versehentlich das falsche Feld ankreuzte, dass er in informellen Gesprächen zwischen den beiden Wahlgängen überzeugt wurde oder dass er durch die Debatte unschlüssig wurde, aber dann das Vertrauen in seine ursprüngliche Präferenz zurückgewann. 
besonders hoch war, während die Anzahl derer, die sich für den Kompromissantrag aussprachen, unter den Nichtunterzeichnern besonders hoch war.

Verglichen mit der Nullhypothese, dass die Stimmen der Nichtunterzeichner ebenso auf die drei Anträge verteilt sind wie die der Unterzeichner, kann der Kompromissantrag einen klaren Zuwachs an Stimmen verzeichnen, während der Verbotsantrag verliert. Ist dies ein Anzeichen dafür, dass die Debatte die Präferenzen in Richtung des Kompromissantrags beeinflusst hat? Obwohl diese Interpretation im Hinblick auf die Tauglichkeit des Parlaments als deliberatives Forum günstig wäre und obwohl sie von Anhängern des Kompromissantrags unterstützt wird, gibt es plausible Alternativerklärungen: Die eine liegt in der Tendenz von Nicht-Experten, einen Mittelweg zu suchen, statt in einer ihnen wenig vertrauten Frage eine extreme Position einzunehmen. Eine andere liegt in dem Umstand, dass sich die Gruppe, die sich für ein vollständiges Verbot aussprach, bereits viel früher und in einem anderen Kontext formiert hatte, als die Gruppe, die den Kompromissantrag entwickelte. Der unerwartete Erfolg des Kompromissantrags im Vergleich mit der Anzahl seiner Unterzeichner ist insofern ein wenig überzeugender Indikator für Präferenztransformation während der Debatte.

Daher, und um mehr über die Präferenztransformation der Parlamentarier herauszufinden, die keinen der Anträge unterschrieben hatten, wurde unter diesen eine anonyme Befragung durchgeführt. Sie sollten angeben, wann sie sich eine Meinung in dieser Angelegenheit gebildet hatten und inwieweit diese von der Debatte beeinflusst wurde. Von den 138 ,unentschiedenen“ Parlamentariern schickten 26 (19 Prozent) den Fragebogen zurück. Angesichts der Tatsache, dass die Entscheidung über den Import embryonaler Stammzellen zu diesem Zeitpunkt beinahe vier Jahre zurücklag, ist die niedrige Antwortrate wenig überraschend. ${ }^{46}$ Die geringe Anzahl der Antwortenden verbietet es, weitreichende Schlüsse aus der Befragung zu ziehen. Dennoch sind einige Extrapolationen möglich und liefern Hinweise im Hinblick auf die Möglichkeit von Präferenztransformation in parlamentarischen Debatten.

Auf die Frage, ob sie sich mit dem Thema im Vorfeld der Bundestagsdebatte befasst hätten, antworten 21 von 26 entweder ,ja, intensiv“ oder ,ja, ein wenig“. Zehn geben an, dass sie zu Beginn der Plenardebatte eine feste Meinung hatten, neun antworten mit „eher ja“ und sieben mit „eher nein“, so dass wirklich unentschlossene Mitglieder eindeutig in der Minderheit sind. Gefragt, ob die Debatte ihnen geholfen hat, offene Fragen zu klären, sagen 16 „nein“ oder „eher nein“. Hinsichtlich der Gründe zeigt sich ein ähnliches Bild: 14 antworten mit „nein“ oder „eher nein“ auf die Frage, ob sie neue Bewertungsaspekte erkannt haben. In diesem Fall geben jedoch sechs Mitglieder eine klar positive Antwort und weitere sechs antworten ,vielleicht“.

Trotz des Umstands, dass sowohl kognitive Einstellungen als auch praktische Gründe nicht gänzlich von Argumenten in der Debatte unbeeinflusst blieben, existiert nur ein einziger Fall von manifester Präferenztransformation in der Stichprobe: Eine Befragte gibt an, dass sie, obwohl sie im Vorhinein das vollständige Verbot unterstützt hatte, überzeugt wurde, für den Kompromissantrag zu stimmen. Sieben Befragte teilen mit, dass sie von einem

46 Um einzuschätzen, ob die Stichprobe repräsentativ für die Gesamtheit ist, wurden die Teilnehmer gebeten, ihr Geschlecht anzugeben und ob sie den Regierungs- oder den Oppositionsfraktionen angehörten. Die Ergebnisse zeigen, dass die Befragten überproportional häufig männlich waren und den Oppositionsfraktionen angehörten. 
anderen Abgeordneten wissen, der seine Präferenz während der Debatte geändert hat, während 19 keinen solchen Fall von Präferenzwandel kennen. Natürlich könnten diejenigen, die angeben, eine Person zu kennen, die ihre Präferenz geändert hat, alle an dieselbe Person denken. Da sie auf Regierungs- und Oppositionsparteien verteilt sind, ist es jedoch wahrscheinlicher, dass einige von ihnen von unterschiedlichen Fällen wissen. Von der Stichprobe auf die Gesamtzahl der Nicht-Unterschreibenden extrapolierend, erscheint es gerechtfertigt anzunehmen, dass höchstens zwischen einem und drei Prozent der Bundestagsmitglieder ihre Präferenz als Ergebnis der Debatte geändert haben.

Die Analyse der Deliberation in der Konsenskonferenz lässt ein größeres Ausmaß und eine andere Richtung der Präferenztransformation erkennen. Eine Mehrheit der Bürger bestätigt einen starken Einfluss der Experten auf ihre Meinungsbildung. ${ }^{47}$ Direkt nach der Anhörung gibt etwa die Hälfte der Teilnehmer an, dass diese sie skeptischer gemacht hat. ${ }^{48}$ Allerdings hatten die Bürger selbst die überwiegend skeptischen Experten ausgewählt, was für eine kritische Grundtendenz in der Gruppe spricht. Außerdem zeigen die Transkripte eine gewisse Entwicklung weg von faktischen, hin zu ethischen und politischen Fragen. Eine Frage des Evaluationsteams betraf die Aspekte, die die Teilnehmer als wichtig für die Bewertung der embryonalen Stammzellforschung erachteten. Während die Reihenfolge der Aspekte in der Kontrollgruppe zwischen den Zeitpunkten des ersten und dritten Konferenzwochenendes stabil blieb, ${ }^{49}$ änderte sie sich in der Bürgergruppe während der drei Wochenenden signifikant. ${ }^{50}$ „Ethische Aspekte“ werden von Beginn an am höchsten bewertet, „soziale“ anfangs sehr niedrig, gewinnen aber schließlich an Bedeutung und „politische Aspekte“, die an den ersten beiden Wochenenden sehr niedrig eingeschätzt wurden, hatten am dritten Wochenende deutlich an Bedeutung zugenommen. „Gesundheitliche Aspekte“, die am ersten Wochenende an zweiter Stelle standen, büßten in der Bürgermeinung deutlich an Relevanz ein, ähnlich wie „ökonomische“. „Religiöse Aspekte“ lagen von Beginn an niedrig, während „wissenschaftliche“ auf einem hohen Niveau stabil blieben.

Der Wandel in als relevant erachteten Bewertungsaspekten verdeutlicht die Veränderungen in der Abwägung und Aggregation praktischer Gründe und insofern die Präferenztransformation auf der volitiven und nicht nur auf der kognitiven Seite. Der Umstand, dass soziale und politische Aspekte im Vergleich zu ökonomischen und gesundheitlichen an Relevanz gewannen, lässt auf eine Politisierung des Forums schließen. Auch wenn „wissenschaftliche Aspekte" noch immer wichtig sind, hat die Bürgergruppe offensichtlich die Rhetorik der „Ethik des Heilens“ durchschaut, mit der Befürworter der Stammzellforschung in Politik und Medien das Potenzial für Heilung mit der eigentlichen Heilung verwechseln. Die Information über den tatsächlichen Stand der Forschung (Grundlagenforschung ohne Anwendungsmöglichkeiten in der näheren Zukunft) und die kritischen Stimmen von Patientenvertretern in der Anhörung lösten zunächst einen Wandel kognitiver Einstellungen aus und veränderten dann die Einschätzung der praktischen Gründe.

Betrachtet man die gesamte Gruppe und vergleicht sie mit der Kontrollgruppe, wird nicht nur deutlich, dass Präferenztransformation tatsächlich stattfand und ihren Ursprung sowohl auf der volitiven als auch auf der kognitiven Seite hatte, sondern auch, dass Präfe-

47 Vgl. Hans Jörg Henning / Birgitt Erdwien, a.a.O. (Fn. 41), S. 64.

48 Vgl. ebenda, S. 57.

49 Vgl. ebenda, S. 26.

50 Vgl. ebenda, S. 25. 
renzen in eine bestimmte Richtung konvergierten: Entgegen den Hoffnungen von Wissenschaftspolitikern wurden die Bürger den neuen Technologien embryonaler Stammzellforschung gegenüber kritischer. ${ }^{51}$

Insgesamt scheinen weder die Bundestagsdebatte noch die Bürgerkonferenz zum Import embryonaler Stammzellen perfekte Institutionalisierungen der Debatte beziehungsweise der Deliberation als jeweilige idealtypische Interaktionsmodi gewesen zu sein. Die parlamentarische Konfiguration hat den Diskurs und die Koordination in diesem Fall anscheinend stärker begünstigt als die idealtypische Debatte. Die Bürgerkonferenz war, obwohl vollständig diskursiv, weniger koordinativ als die idealtypische Deliberation. Nichtsdestoweniger wurden die Hypothesen über Interaktionsmodi und Präferenztransformation für diese zwei Einzelfälle bestätigt.

\section{Fazit: Politik hat mehr als einen Ort}

Theoretisches Modell und empirische Analyse unterstreichen eine zentrale Annahme in der Theorie deliberativer Demokratie, nämlich dass Präferenztransformation nur im Interaktionsmodus der Deliberation wahrscheinlich ist. Wir kommen jedoch zu dem Schluss, dass jenes Forum, das traditionell als die Institutionalisierung von Deliberation gilt - die Plenarsitzung in der parlamentarischen Demokratie - sowohl die Deliberation als dominanten Interaktionsmodus (zumindest unserer Definition nach) als auch Präferenztransformation tendenziell unmöglich macht. Dies bedeutet aber nicht, dass Plenardebatten keine maßgebliche Rolle in der Demokratie spielen. Auch Debatten zur Erklärung von Entscheidungen ${ }^{52}$ dienen der Öffentlichkeitsfunktion des Parlaments. Zudem finden im Bundestag auch andere Formen kommunikativer Interaktion statt: So sind etwa Große Anfragen, Aktuelle Stunden oder die Erste Lesung von Gesetzentwürfen von stärkeren deliberativen Elementen geprägt, da sie nicht oder noch nicht dem unmittelbaren Druck der Mehrheitsfindung unterliegen. ${ }^{53}$

Die unterschiedlichen Rollen der verschiedenen Interaktionsmodi müssen daher in Entscheidungsprozessen berücksichtigt werden. Angesichts der Tatsache, dass Deliberation offenkundig schwer zu institutionalisieren ist, sollten Anhänger der deliberativen Demokratietheorie und andere Forscher, die sich für das Zustandekommen politischer Entscheidungen interessieren, der Rolle informeller Gespräche von Bürgern über Politik und den Beziehungen zwischen Mediendebatten, öffentlicher Meinung und formellen Institutionen mehr Aufmerksamkeit entgegenbringen. Nichtsdestoweniger können partizipative Verfahren zur Belebung der Demokratie beitragen und sollten keinesfalls aufgegeben werden.

Ebenfalls sollte in partizipativen Experimenten am Ziel einer expliziten Entscheidung, wenn nicht sogar eines Konsenses, festgehalten und die politische Natur des Konflikts in den jeweiligen Foren und Verfahren anerkannt werden. Viele der bestehenden Modelle erscheinen nicht koordinativ genug und führen deshalb zu einer Form der Interaktion, die

51 Vgl. Birgitt Erdwien, Bürgerpartizipation als Gruppenprozess: Ergebnisse der begleitenden Evaluation, in: Christof Tannert / Peter Wiedemann (Hrsg.), a.a.O. (Fn. 44), S. 130 - 137, S. 136.

52 Vgl. Wolfgang Zeh, a.a.O. (Fn. 18), S. 923.

53 Vgl. ebenda, S. 929 f. 
dem Modus der Diskussion näher steht als dem der Deliberation. Da möglicherweise eine Form von Trade-Off zwischen Koordinativität und Diskursivität besteht, dürfte es sinnvoll sein, sich hier eher in die Richtung des Verhandelns zu bewegen, etwa mithilfe von Modellen wie der Mediation. Dies mag bedeuten, dass auch nicht-generalisierbare Gründe und Interessen in die Kommunikation einfließen, würde aber die Notwendigkeit von Kompromissen zwischen fundamentalen Werten und unüberwindbar widersprüchlichen Interessen verdeutlichen. Allgemeiner betrachtet sind wir der Ansicht, dass die Rolle des Kompromisses in der Politik eine positive Neubewertung verdient.

In der empirischen Deliberationsforschung ist der systematische Vergleich verschiedener Modelle deliberativer Bürgerbeteiligung ein Desiderat. Ein weiterer Ansatz könnte darin bestehen, Bürgerforen ähnlichen Aufbaus, die unterschiedliche Konflikte behandeln, zu vergleichen. ${ }^{54}$ Vielen experimentellen Ansätzen, die mit Sicherheit in sich wertvoll sind, fehlt diese Art der Variation auf der unabhängigen Variablen, was dazu führt, dass ein erhebliches Erkenntnispotenzial unausgeschöpft bleibt.

54 Ein aktueller Aufsatz von Carolyn M. Hendriks / John Dryzek / Christian Hunold entwickelt in dieser Richtung einen interessanten Ansatz; dies., Turning up the Heat. Partisanship in Deliberative Innovation, in: Political Studies, 55. Jg. (2009), H. 2, S. 362 - 383. In Claudia Landwehr, a.a.O. (Fn. 14), wird die hier dargestellte Unterscheidung idealtypischer Interaktionsmodi mit ihrer jeweiligen Institutionalisierung in einem internationalen Vergleich von Gremien, die über die Verteilung von Gesundheitsgütern entscheiden, angewandt. 